\title{
Confining Solution of the Dyson-Schwinger Equations in Coulomb Gauge
}

\author{
D. Epple, H. Reinhardt, W. Schleifenbaum \\ Institut für Theoretische Physik \\ Auf der Morgenstelle 14 \\ D-72076 Tübingen \\ Germany
}

\begin{abstract}
The Dyson-Schwinger equations arising from minimizing the vacuum energy density in the Hamiltonian approach to Yang-Mills theory in Coulomb gauge are solved numerically. A new solution is presented which gives rise to a strictly linearly rising static quark potential and whose existence was previously observed in the infrared analysis of the Dyson-Schwinger equations. For the new solution we also present the static quark potential and calculate the running coupling constant from the ghost-gluon vertex.
\end{abstract}

PACS numbers: 12.38.Aw, 14.70.Dj, 12.38.Lg, 11.10.Ef 


\section{INTRODUCTION}

Recently, there has been renewed interest in the Hamiltonian approach to Yang-Mills theory in Coulomb gauge [1]. In this gauge the Yang-Mills Schrödinger equation was solved variationally in the continuum using Gaussian types of wave functionals [2, 3, 4]. Minimization of the energy density results in a set of Dyson-Schwinger equations. These equations have been solved analytically in the infrared using power law ansätze for the corresponding Green functions [5]. Thereby, two infrared solutions of the Dyson-Schwinger equations have been found which differ in the infrared exponents. Only one of these two solutions was previously found in the numerical solution of the Dyson-Schwinger equations in the whole momentum regime [3]. We present here the other one which has the attractive property that it gives rise to a strictly linear rising static quark potential.

The organization of the paper is as follows: In the next section we briefly summarize the basic ingredients of the Hamiltonian approach to Yang-Mills theory in Coulomb gauge and present the resulting Dyson-Schwinger equations. The numerical solutions of these equations are presented in section 3. We also calculate from these solutions the static quark potential and the running coupling constant. Finally, our conclusions are given in section 4 .

\section{THE DYSON-SCHWINGER EQUATIONS IN THE HAMILTONIAN AP- PROACH TO YANG-MILLS THEORY IN COULOMB GAUGE}

In Coulomb gauge, where $\vec{\partial} \cdot \vec{A}=0$, the Yang-Mills Hamiltonian reads [1]

$$
H=\frac{1}{2} \int J^{-1} \Pi J \Pi+\frac{1}{2} \int B^{2}+\frac{g^{2}}{2} \int J^{-1} \rho(-\hat{D} \partial)^{-1}\left(-\partial^{2}\right)(-\hat{D} \partial)^{-1} J \rho .
$$

Here, $\Pi_{i}^{a}(x)=\delta / i \delta A_{i}^{a}(x)$ denotes the momentum operator, representing the color electric field, $B$ is the color magnetic field and $\rho^{a}(x)=-\hat{A}_{i}^{a b}(x) \Pi_{i}^{b}(x)$ is the non-Abelian color charge of the gauge field. Furthermore, $J(A)=\operatorname{Det}\left(-\hat{D}_{i} \partial_{i}\right)$ is the Faddeev-Popov determinant with $\hat{D}_{i}^{a b}=\delta^{a b} \partial_{i}+\hat{A}_{i}^{a b}$ being the covariant derivative in the adjoint representation of the gauge group $\left(\hat{A}_{i}^{a b}=f^{a c b} A_{i}^{c}\right.$ with the structure constants $\left.f^{a b c}\right)$. The first term in the Hamiltonian is the Laplacian in the curved space of gauge orbits, the second term represents the potential and the last term arises from the longitudinal momentum part of the kinetic energy after resolving Gauss' law. We use the following ansatz for the vacuum wave functional [3]

$$
\Psi[A]=\mathcal{N} \frac{1}{\sqrt{J[A]}} \exp \left(-\frac{1}{2} \int A \omega A\right),
$$

where $\omega\left(\left|x-x^{\prime}\right|\right)$ is a variational kernel determined by minimizing the energy

$$
\langle\Psi|H| \Psi\rangle=\int \mathcal{D} A J[A] \Psi^{*}[A] H \Psi[A] .
$$

This yields a set of coupled Dyson-Schwinger equations for the ghost propagator

$$
G^{a b}\left(x, x^{\prime}\right)=\left\langle\Psi\left|\left\langle x, a\left|(-\hat{D} \partial)^{-1}\right| x^{\prime}, b\right\rangle\right| \Psi\right\rangle=\frac{1}{g}\left(-\partial^{2}\right)^{-1} d\left(x, x^{\prime}\right),
$$


the gluon propagator

$$
D_{i j}^{a b}\left(x, x^{\prime}\right)=\left\langle\Psi\left|A_{i}^{a}(x) A_{j}^{b}\left(x^{\prime}\right)\right| \Psi\right\rangle=\frac{1}{2} \delta^{a b} t_{i j}(x) \omega^{-1}\left(x, x^{\prime}\right), t_{i j}(x)=\delta_{i j}-\frac{\partial_{i}^{x} \partial_{j}^{x}}{\partial_{x}^{2}},
$$

and the Coulomb form factor $f$ defined by

$$
\left\langle\Psi\left|(-\hat{D} \partial)^{-1}\left(-\partial^{2}\right)(-\hat{D} \partial)^{-1}\right| \Psi\right\rangle=\left\langle\Psi\left|(-\hat{D} \partial)^{-1}\right| \Psi\right\rangle\left(-\partial^{2}\right) f\left\langle\Psi\left|(-\hat{D} \partial)^{-1}\right| \Psi\right\rangle \text {. }
$$

A further quantity arising in the evaluation of $\langle\Psi|H| \Psi\rangle$ is the scalar curvature $\chi$,

$$
\chi\left(x, x^{\prime}\right)=\frac{1}{2\left(N_{C}^{2}-1\right)} t_{i j}(x) \chi_{i j}^{a a}\left(x, x^{\prime}\right), \quad \chi_{i j}^{a b}\left(x, x^{\prime}\right)=-\frac{1}{2}\left\langle\Psi\left|\frac{\delta^{2} \ln J[A]}{\delta A_{i}^{a}(x) \delta A_{j}^{b}\left(x^{\prime}\right)}\right| \Psi\right\rangle,
$$

which denotes the ghost loop contribution to the gluon energy. This quantity measures the "curvature" of the space of transversal gauge orbits and vanishes in QED.

Following ref. [3], we will solve here the Dyson-Schwinger equations to one-loop order. To this order it is sufficient to calculate the Coulomb form factor $f$ perturbatively to ensure the proper ultraviolet asymptotics of the corresponding loop integrals (see ref. [3] for more details). In the infrared, this results in $f(k \rightarrow 0)=$ const. After regularization and renormalization using subtracted equations with some arbitrary renormalization scale $\mu$, the remaining Dyson-Schwinger equations for the ghost and gluon propagators and the expression for the curvature in momentum space read [3]

$$
\begin{aligned}
d^{-1}(k) & =d^{-1}(\mu)-\Delta I_{d}(k, \mu), \\
\omega^{2}(k) & =\chi^{2}(k)+k^{2}+2 \chi(k)\left(\Delta I_{\omega}^{(1)}(k, \mu)-\Delta I_{\omega}^{(1)}(0, \mu)+c\right)+\Delta I_{\omega}^{(2)}(k, \mu)+c_{0}, \\
\chi(k) & =\chi(\mu)+\Delta I_{\chi}(k, \mu),
\end{aligned}
$$

with $c_{0}=\omega^{2}(\mu)-\chi^{2}(\mu)-\mu^{2}-2 \chi(\mu)\left(-\Delta I_{\omega}^{(1)}(0)+c\right)$. Here $d^{-1}(\mu), \omega(\mu), \chi(\mu)$, and $c$ are renormalization constants. Furthermore, the $\Delta I_{x}$ refer to the following differences of loop integrals

$$
\begin{aligned}
\Delta I_{d}(k, \mu) & =I_{d}(k)-I_{d}(\mu), I_{d}(k)=\frac{N_{c}}{2} \int \frac{d^{3} q}{(2 \pi)^{3}}\left(1-(\hat{\mathbf{k}} \cdot \hat{\mathbf{q}})^{2}\right) \frac{d(|\mathbf{k}-\mathbf{q}|)}{(\mathbf{k}-\mathbf{q})^{2} \omega(q)} \\
\Delta I_{\chi}(k, \mu)= & I_{\chi}(k)-I_{\chi}(\mu), I_{\chi}(k)=\frac{N_{c}}{4} \int \frac{d^{3} q}{(2 \pi)^{3}}\left(1-(\hat{\mathbf{k}} \cdot \hat{\mathbf{q}})^{2}\right) \frac{d(|\mathbf{k}-\mathbf{q}|) d(q)}{(\mathbf{k}-\mathbf{q})^{2}} \\
\Delta I_{\omega}^{(n)}(k, \mu)= & I_{\omega}^{(n)}(k)-I_{\omega}^{(n)}(\mu), n=1,2 \\
I_{\omega}^{(n)}(k)= & \frac{N_{c}}{4} \int \frac{d^{3} q}{(2 \pi)^{3}}\left(1+(\hat{\mathbf{k}} \cdot \hat{\mathbf{q}})^{2}\right) \\
& \frac{d(|\mathbf{k}-\mathbf{q}|)^{2} f(|\mathbf{k}-\mathbf{q}|)}{(\mathbf{k}-\mathbf{q})^{2}} \cdot \frac{[\omega(q)-\chi(q)]^{n}-[\omega(k)-\chi(k)]^{n}}{\omega(q)} .
\end{aligned}
$$

For the solution of the coupled Dyson-Schwinger equations (8) and (9), we implement the horizon condition [6]

$$
d^{-1}(\mu=0)=0 \text {. }
$$

Assuming power law behaviour for the Green functions in the infrared

$$
\omega(k)=\frac{A}{k^{\alpha}}, d(k)=\frac{B}{k^{\beta}},
$$


the infrared analysis of the ghost Dyson-Schwinger equation yields the sum rule

$$
2 \beta=\alpha+1,
$$

due to the non-renormalization of the ghost-gluon vertex, a feature of both Landau [7] and Coulomb gauge [5, 8]. The horizon condition (14) implies $\beta>0$. For $\beta>1 / 2$ we obviously obtain an infrared divergent gluon energy which is a manifestation of confinement. Furthermore, $\beta>1 / 2$ also implies an infrared divergent curvature $\chi(k)$. Indeed, an infrared analysis [5] of Eq. (12) shows that for $k \rightarrow 0, \chi(k) \propto k^{2 \beta-1}$ which by the sum rule (16) is the infrared behaviour of $\omega(k)$. This is consistent with the gap equation (9)). For an infrared divergent $\chi(k)$ the gap equation (9) reduces in the infrared to

$$
\lim _{k \rightarrow 0}(\omega(k)-\chi(k))=c,
$$

implying that $\omega(k)$ and $\chi(k)$ have the same infrared (divergent) behaviour. Eq. (17) along with the ghost Dyson-Schwinger equation (8) in the infrared limit can be solved analytically for the infrared exponents. It was found earlier that, using angular approximation, one finds only one solution $\beta=1[3]$. However, performing an infrared analysis without angular approximation, there are the two solutions $[5]$

$$
\beta_{1} \approx 0.796, \quad \beta_{2}=1 .
$$

A numerical solution of the set of equations without the angular approximation was presented in ref. [3] corresponding to the value $\beta_{1} \approx 0.796$. In this paper we present an improved numerical method with which the infrared exponent $\beta_{2}=1$ is found as well.

After obtaining the self-consistent solutions for the form factors numerically, we may apply them to the calculation of the static quark potential on the one hand, and a non-perturbative running coupling on the other. The static quark potential $V(r)$ can be computed by taking the expectation value of the Coulomb term of the Yang-Mills Hamiltonian (11) where $\rho$ is chosen to be the charge distribution of two heavy color charges, i.e.

$$
\rho(x)=\delta^{(3)}(x-r / 2)-\delta^{(3)}(x+r / 2) .
$$

After Fourier transform, we arrive at

$$
V(r)=\int \frac{d^{3} q}{(2 \pi)^{3}} \frac{d^{2}(q) f(q)}{q^{2}}\left(1-e^{i \mathbf{k} \cdot \mathbf{r}}\right) .
$$

The solution with $\beta_{2}=1$, see Eq. (18), has the attractive feature that it gives rise to a strictly linearly rising static quark potential $V(r)=\sigma r$.

A non-perturbative running coupling may be extracted from the $\mathrm{RG}$ invariant $V(r)$ given in Eq. (20) and would be divergent in the infrared. At the same time, it is possible to define the running coupling via the ghost-gluon vertex [8],

$$
\alpha(k)=\frac{16}{3} \frac{g_{r}^{2}}{4 \pi} k^{5} G^{2}(k) D(k)=\frac{2}{3 \pi} k d^{2}(k) \omega^{-1}(k),
$$

and find that, due to the non-renormalization of the ghost-gluon vertex, $\alpha(k)$ has an infrared fixed point. This can be seen immediately when plugging in the sum rule (16) into Eq. (21). It was shown in ref. [5] that $\alpha(0)=\frac{16 \pi}{3 N_{c}}$ for $\beta=\beta_{2}$ by infrared analysis of the Dyson-Schwinger equations. The same value is actually found when resorting to the angular approximation as done in [3]. There, it was shown that $\frac{A}{B^{2}}=\frac{N_{c}}{6 \pi^{2}} \frac{\beta+2}{2 \beta(\beta+1)}$ which yields the above mentioned infrared fixed point if $\beta=\beta_{2}$. 


\section{NUMERICAL SOLUTION OF THE DYSON-SCHWINGER EQUATIONS}

In order to solve the set of integral equations (8) and (9) numerically, we use a Chebyshev approximation on a logarithmic scale for the form factors. A logarithmic momentum scale is introduced to have enough nodes at every order of magnitude. Furthermore, infrared- or ultraviolet divergent functions are expressed on a double-logarithmic scale.

Whenever we encounter a change of sign in one of the functions, the logarithmic scale demands a separate treatment of positive and negative values. In particular, the function $\chi(k)$ goes to $+\infty$ for $k \rightarrow 0$ and to $-\infty$ for $k \rightarrow \infty$, with a change of sign at some intermediate point which we can fix by the renormalization condition $\chi(\mu)=0$. This knowledge then allows us to fit $\chi(k)$ logarithmically in the infrared, and to fit $-\chi(k)$ also logarithmically in the ultraviolet. For some range around $k=\mu$, we fit $\chi(k)$ linearly. The resulting set of nodes is to be used also for the other form factors. This is done to minimize the number of required Chebyshev evaluations since every evaluation of a fit is subject to some non-zero error.

For asymptotic values of momentum, it is instructive for the numerics to make use of the analytical results, cf. the methods used in ref. [9]. The set of nodes cover a finite momentum region. In order to extrapolate this to the whole infinite momentum range, the general algebraic forms obtained analytically serve as ansätze. However, contrary to what has been previously done in Dyson-Schwinger studies, the parameters of these asymptotic forms are still determined numerically. E.g., in the infrared it was shown analytically that power laws provide a solution. The numerics will have the asymptotic form of the power laws (15) as an input but determine the various exponents and coefficients as parameters by nonlinearleast-squares fitting. Thus, this numerical method provides a check on the analytical results (18) rather than imposing the latter.

Furthermore, since the set of integral equations comprises the difference $\omega(k)-\chi(k)$ for small momenta $k$, we extract the infrared power law $A / k^{\alpha}$ of $\omega(k)$ and $\chi(k)$ in a single fit with the combined data which gives us $A$ and $\alpha$, and extract the next-to-leading order constants $\omega_{0}$ and $\chi_{0}$, again with least-squares fits. This gives us fits for $\omega(k)$ and $\chi(k)$ up to intermediate momentum values,

$$
\omega(k)=\frac{A}{k^{\alpha}}+\omega_{0}, \quad \chi(k)=\frac{A}{k^{\alpha}}+\chi_{0}, \quad k \rightarrow 0 .
$$

It is possible to gain more information on the intermediate momentum regime by setting up an infrared expansion of the function (cf. Eq. (17))

$$
\nu(k):=\omega(k)-\chi(k)=c+c_{1} k^{\gamma}, \quad k \rightarrow 0, \gamma>0 .
$$

and determining its parameters by least-squares fitting. However, this works only if the form factors already are pretty close to the (yet unknown) solution. Typically, at intermediate iteration stages $\nu(k)$ is an infrared divergent quantity (as opposed to the fully iterated solutions). It is then mandatory to control that the fit of the function $\nu(k)$ converges to a sensible value at each iteration step.

Ultraviolet extrapolation is done in a similar way. We know the asymptotic behaviour from the UV analysis, see ref. [3], and make corresponding ansätze for the form factors in the UV,

$$
\begin{array}{ll}
\omega(k \rightarrow \infty)=k, & \chi(k \rightarrow \infty) \propto k / \sqrt{\ln \left(k / \mu_{\chi}\right)}, \\
d(k \rightarrow \infty) \propto 1 / \sqrt{\ln \left(k / \mu_{d}\right)}, & f(k \rightarrow \infty) \propto 1 / \sqrt{\ln \left(k / \mu_{f}\right)},
\end{array}
$$



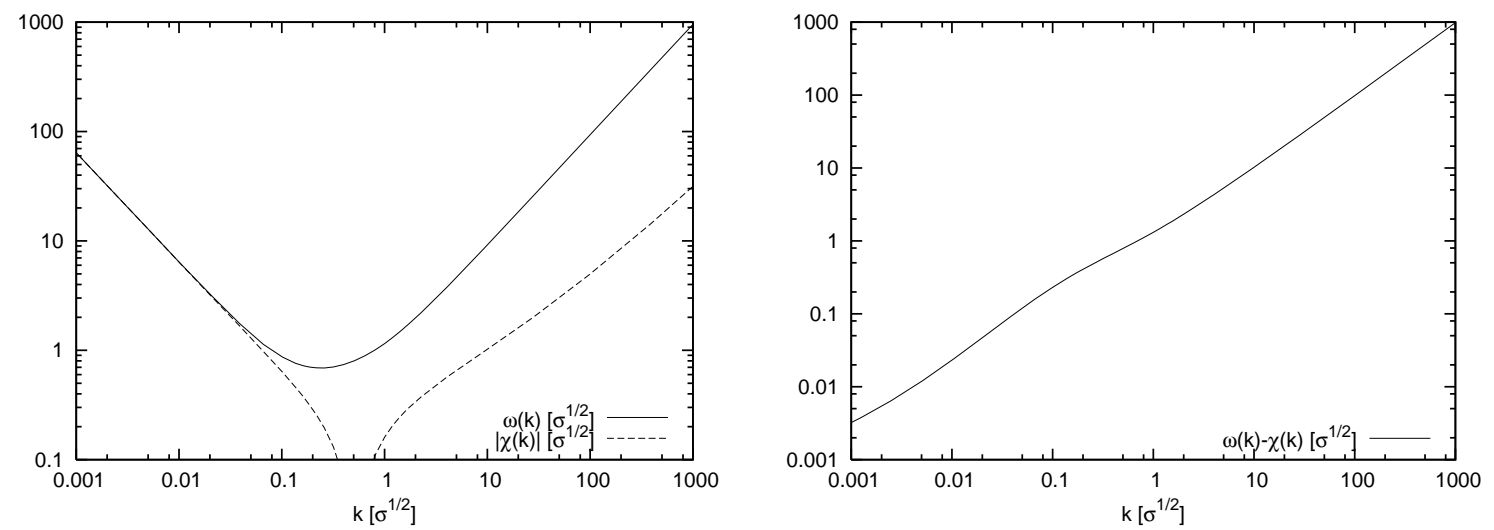

FIG. 1: Left panel: the gluon energy $\omega(k)$ and the modulus of the scalar curvature $\chi(k)$. Right panel: the difference $\omega(k)-\chi(k)$.

extracting the coefficients as well as the different scale parameters $\mu_{\chi}, \mu_{d}, \mu_{f}$ using leastsquares fitting.

Equipped with the discretized form factor ansätze, we can now perform the numerical integration by quadrature followed by iteration to determine the fit parameters. Here, we use the set of renormalized and thus finite integral equations (8) and (9). Whereas the angular integrals are performed with the customary Gauss-Legendre formula, the radial integration involves additional non-linear transformations. Having introduced finite infrared and ultraviolet cutoffs $\varepsilon$ and $\Lambda$, resp., for the radial integrals of the kind $\int_{0}^{\infty} d x f(x),{ }^{1}$ we transform the integration variable logarithmically to map the integration interval to $[\ln \varepsilon, \ln \Lambda]$. Like in the Chebyshev expansion, this logarithmic representation is chosen to have enough nodes at every order of magnitude. The remaining integral over this interval is evaluated numerically using a Gauss-Legendre algorithm.

The numerical solution is then carried out iteratively, thereby using some relaxation method (like $\left.x_{\text {new }} \leftarrow r x_{\text {new }}+(1-r) x_{\text {old }}\right)$ to compute the updated values for the form factors at the Chebyshev nodes. For a stable iteration we use $r \approx 0.1$. These new values are then used to extract the Chebyshev coefficients and the fit parameters for the infrared and ultraviolet extrapolations. This gives us new form factors.

For the explicit calculation it is convenient to express the Dyson-Schwinger equations in terms of dimensionless quantities by rescaling all dimensionful quantities with appropriate powers of the renormalization scale $\mu$. Indicating the rescaled quantities by bars, one has

$$
\begin{aligned}
\bar{k}=\frac{k}{\mu}, & \bar{\omega}(\bar{k})=\frac{\omega(k=\mu \bar{k})}{\mu}, & \bar{d}(\bar{k}) & =d(k=\mu \bar{k}), \\
\bar{\chi}(\bar{k}) & =\frac{\chi(k=\mu \bar{k})}{\mu}, & \bar{c} & =\frac{c}{\mu} .
\end{aligned}
$$

Furthermore, we have to specify the renormalization constants, which are $\bar{d}(1), \bar{\omega}(1), \bar{\chi}(1)$, $\bar{c}$. It was already observed in ref. [3] that neither the infrared exponents nor the ultraviolet

\footnotetext{
${ }^{1}$ The error thus involved can be shown to be of the order $\mathcal{O}(\varepsilon)$ and $\mathcal{O}(1 / \Lambda)$ to some positive power. Choosing $\varepsilon$ sufficiently small and $\Lambda$ sufficiently large will render the error negligible.
} 

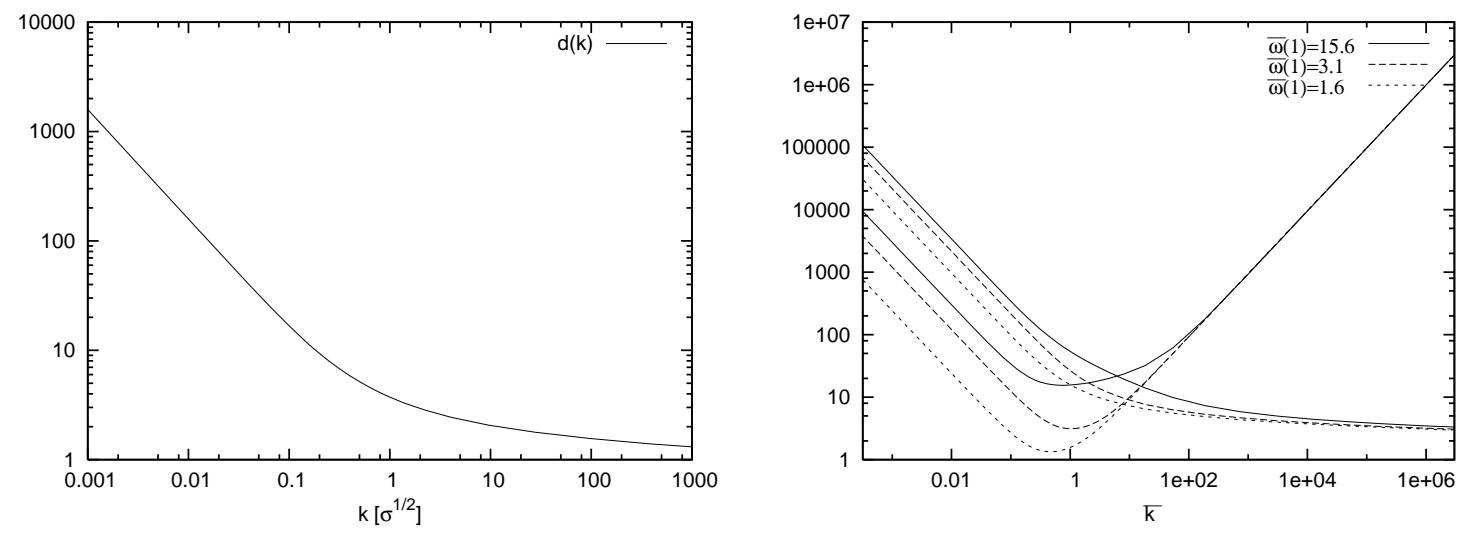

FIG. 2: Left panel: $d(k)$. Right panel: the functions $\omega(k)$ and $d(k)$ for different values of $\omega(\mu)$.

behaviour of the various form factors depend on the precise value of the renormalization constants except for the value of $\bar{d}(1)$ which is, however, fixed by the horizon condition $d^{-1}(0)=0$. The remaining renormalization constants are fixed as follows. Throughout our calculations we choose $\bar{\chi}(1)=0$. This choice is possible since from the infrared and ultraviolet analysis of the Dyson-Schwinger equations carried out in ref. [3] follows that $\chi(k)$ has a zero at some finite momentum value. Choosing $\bar{\chi}(1)=0$ has the advantage that the renormalization of the curvature $\chi(k)$ just removes the ultraviolet divergence without introducing any new finite parameter. In the same spirit we chose $c=0$ which implies that $\omega(k)$ and $\chi(k)$ approach each other for $k \rightarrow 0$ (see Eq. (17)). This choice is preferred by the study of the 't Hooft loop [10, 11]. The remaining renormalization constant $\bar{\omega}(1)$ is considered as a parameter.

Figures 1 and 2 show the numerical solution of the coupled Dyson-Schwinger equations for the choice $\bar{\omega}(1)=1.6$. As seen from the left panel in Fig. 1, $\omega(k)$ and $\chi(k)$ indeed obey the same infrared divergent behaviour, in agreement with the infrared analysis of the DysonSchwinger equations [5]. Furthermore from the right panel of Fig. 1 we also notice that in the infrared $\omega(k)$ and $\chi(k)$ coincide, in accord with our choice of the renormalization condition $c=0$.

Figure2 shows the dependency of $\omega(k)$ and $d(k)$ upon the renormalization constant $\omega(\mu)$. We find that this constant does not change the infrared exponents and the ultraviolet behaviour but does have impact on the prefactors $A, B$ of the power laws for $\omega(k), \chi(k)$ and $d(k)$. Also, there are some quantitative changes in the intermediate momentum range.

Figure 3 (left panel) shows the running coupling, as defined by Eq. (21). It has an infrared fixed point which is in excellent agreement with the value predicted by the infrared analysis given in [5]. It is also worth mentioning that the running coupling in Fig. 3 is a monotonic function and has no more than one inflection point and thus does not bring up peculiarities in the beta function.

In the right panel of Figure 3 we show the Coulomb potential, as given by Eq. (20). We find an exactly linearly rising behaviour (within an estimated error of less than one percent), again in agreement with the infrared analysis [5]. The linearly rising potential allows us to fit our scale from the string tension $\sigma$. Lattice calculations [12] show, however, that the Coulomb string tension is about a factor of $1.5 \ldots 3$ larger than the string tension extracted from the Wilson loop, in agreement with the analytic result [13] that the Coulomb string 

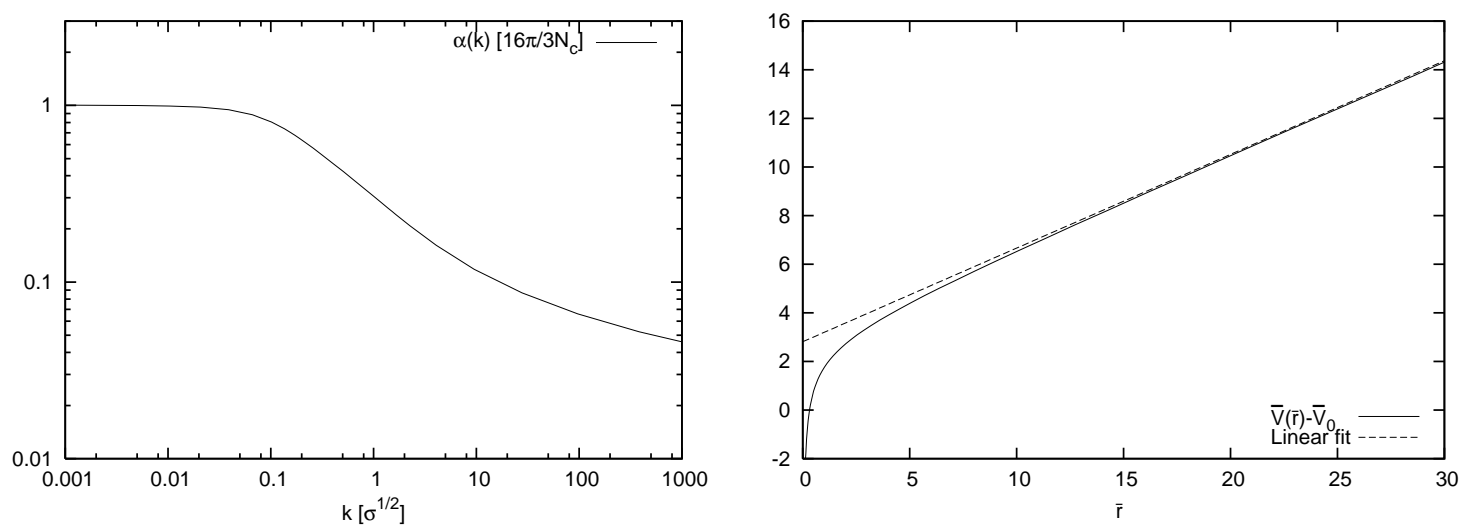

FIG. 3: (left) The running coupling $\alpha(k)$. (right) The Coulomb potential $V(r)$.

tension is an upper bound to the Wilson loop string tension.

\section{SUMMARY AND CONCLUSIONS}

We have numerically solved the Dyson-Schwinger equations following from the minimization of the vacuum expectation value of the Yang-Mills Hamiltonian in Coulomb gauge assuming the horizon condition. The solution found produces a strictly linearly rising static quark potential which is in agreement with the analytic infrared analysis carried out in ref. [5]. We find only a rather weak dependence of our solutions on the specific choice of the remaining renormalization constants, in particular, the infrared exponents and the ultraviolet behaviour are independent of the choices of the renormalization constants, once the horizon condition is adopted. There are, however, changes in the intermediate momentum range which, as will be shown in ref. [10], are tested by the 't Hooft loop.

\section{Acknowledgments}

It is a pleasure to thank A. Szczepaniak for valuable discussions and ongoing correspondence on the subject. We are grateful to the referee for pointing out ref. [9] to us. This work was supported by the Deutsche Forschungsgemeinschaft (DFG) under contract no. Re856/6-1,2 and by the Europäisches Graduiertenkolleg Basel-Graz-Tübingen (EUROGRAD).

[1] N. H. Christ and T. D. Lee, Phys. Rev. D 22 (1980) 939 [Phys. Scripta 23 (1981) 970].

[2] A. P. Szczepaniak and E. S. Swanson, Phys. Rev. D 65 (2001) 025012 arXiv:hep-ph/0107078];

A. P. Szczepaniak, Phys. Rev. D 69 (2004) 074031 [arXiv:hep-ph/0306030.

[3] C. Feuchter and H. Reinhardt, Phys. Rev. D 70 (2004) 105021 arXiv:hep-th/0408236].

[4] H. Reinhardt and C. Feuchter, Phys. Rev. D 71 (2005) 105002 arXiv:hep-th/0408237.

[5] W. Schleifenbaum, M. Leder and H. Reinhardt, Phys. Rev. D 73 (2006) 125019 arXiv:hep-th/0605115]. 
[6] D. Zwanziger, Nucl. Phys. B 485 (1997) 185 arXiv:hep-th/9603203.

[7] J. C. Taylor, Nucl. Phys. B 33 (1971) 436; A. Cucchieri, T. Mendes and A. Mihara, JHEP 0412 (2004) 012 arXiv:hep-lat/0408034]; W. Schleifenbaum, A. Maas, J. Wambach and R. Alkofer, Phys. Rev. D 72 (2005) 014017 arXiv:hep-ph/0411052.

[8] C. S. Fischer and D. Zwanziger, Phys. Rev. D 72 (2005) 054005 arXiv:hep-ph/0504244.

[9] D. Atkinson and J. C. R. Bloch, Phys. Rev. D 58 (1998) 094036 arXiv:hep-ph/9712459]; J. C. R. Bloch, arXiv:hep-ph/0208074; C. S. Fischer and R. Alkofer, Phys. Lett. B 536 (2002) 177 arXiv:hep-ph/0202202] A. Maas, Comput. Phys. Commun. 175 (2006) 167 arXiv:hep-ph/0504110.

[10] H. Reinhardt and D. Epple, to be published.

[11] H. Reinhardt, D. Epple and W. Schleifenbaum, arXiv:hep-th/0610324.

[12] A. Cucchieri and D. Zwanziger, Nucl. Phys. Proc. Suppl. 119 (2003) 727 arXiv:hep-lat/0209068; J. Greensite and S. Olejnik, Phys. Rev. D 67 (2003) 094503 arXiv:hep-lat/0302018; K. Langfeld and L. Moyaerts, Phys. Rev. D 70 (2004) 074507 arXiv:hep-lat/0406024.

[13] D. Zwanziger, Phys. Rev. Lett. 90 (2003) 102001 arXiv:hep-lat/0209105. 